\title{
Vector Equilibrium Problem in Topological Vector Spaces
}

\section{Zhiyong Wu}

Ahead Institute of Software and Technology, Nanchang 330041, China

Keywords: Vector variational inequality; Topological vector space; Existence theorem

\begin{abstract}
In this paper, the vector equilibrium problem in topological vecror spaces is discussed. By using the well-known Ky Fan section theorem, its existence theorems of solution are proved and the main results of reference [3][4] are generalized.
\end{abstract}

Equilibrium problem is 1994 year by mathematician Blum and Oetlli [6] the was explicitly presented for the first time. Because it contains variational inequalities, phase complement problem, fixed point problem, best approximation problem and the Nash equilibrium problem and optimization problem in economics are the special cases, so much attention is paid by many mathematics workers, the equilibrium problem of vector-valued function is a research problem which is more concerned at present. (See Research anthology [5])

\section{Preliminary Knowledge} met:

Definition 1.1 ([1]) Set $\mathrm{X}^{\neq} \phi$, $\tau$ is $\mathrm{X}$ the set family of subsets in if the following properties are (I)X, $\phi \in \tau$;

(II) $\tau$ the collection of any number of subsets in the $\tau$,namely: if $\forall \alpha \in I, G_{\alpha} \in \tau$, 则 $\bigcup_{\alpha \in I} G_{\alpha} \in \tau$;

(III) $\tau$ the intersection of any finite subset of the $\tau$, namely: if $\mathrm{G}^{1}, \mathrm{G}^{2} \ldots, \mathrm{G}^{m} \in \tau, \bigcap_{i=1}^{m} G_{i} \in \tau$; is said $\tau$ is $X$ a topology, which is called $(X, \tau)$ is a topological space.

Definition 1.2 ([1]) $\mathrm{X}$ is a number field $\mathrm{R}$ The linear space on the, $\tau$ is $\mathrm{X}$ a topology on the. if $(\mathrm{X}, \tau)$ satisfies the following conditions:

(1) $\mathrm{X}$ The addition operation in the is continuous;

(2)X the number multiplication operation in the is continuous;

The $\left(X,{ }^{\tau}\right)$ is a topological linear space.

Definition $1.3 \mathrm{X}$ is linear space, $\mathrm{C} \subset \mathrm{X}$, said $\mathrm{C}$ for a cone ,if $\forall x \in \mathrm{C}, \forall \lambda \geq 0$, Yes $\lambda x \in \mathrm{C}$;

If $C$ is a cone and is a convex set, it is said $C$ is a convex cone; if $C \cap(-C)=\{0\}$, Cone $C$ the is the point cone.

Set $\mathrm{P}$ solid topological linear space $\mathrm{Z}$ a closed convex cone in, which $\operatorname{intP} \neq \phi$; $\mathrm{K}$ solid topological linear space $\mathrm{X}$ Non-empty collection in; $f: K \times K \rightarrow Z$ the so-called vector equalization problem ( denoted to VEP) is: Find $x \in K$, Meet (VEP) $f(x, y) \notin$-intP, $\forall y \in K$.

Definition 1.4 Set $\mathrm{X}$ is a topological linear space, $f: X \rightarrow R$, Said $f$ at the point $x \in K$, on the upper half continuous, if $\varlimsup_{y \rightarrow x} f(y) \leq f(x)$; $f$ in $X$ top half Continuous, if $f$ in $X$ each point of the is half continuous; $f$ at the point $x \in K$ for the next half continuous, if $f$ at each point is the upper half continuous; $f$ in $\mathrm{X}$ on the bottom half of the row, if $f$ in $\mathrm{X}$ each point of the is the lower half continuous.

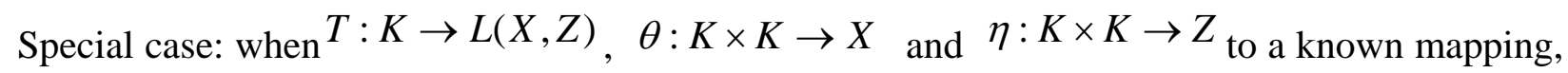


take $f(x, y)_{=<} T x, \theta(y, x)_{>+} \eta(x, y)$, which $L(X, Z)$ represents $\mathrm{X}$ to $\mathrm{Z}$ the space formed by the continuity operator of the, $<l, x>$ expression operator $l \in L(X, Z)$ at the point $x \in X$ the value of the , the vector equalization problem above becomes a generalized variational inequality , that is: ask $x \in X$ meet ( GVVI) $<T x, \theta(y, x)_{>+} \eta(x, y) \notin$-int $\mathrm{P}, \forall y \in K$.

Definition 1.5 A convex subset of set $\mathrm{K}$ to $\mathrm{X}, f: K \rightarrow Z$.If the $\forall x_{1}, x_{2} \in \mathrm{K}$,and $\lambda \in(0,1), \lambda$ $f\left(x_{1}\right)+\left(1-\lambda f\left(x_{2}\right) \in f\left(\lambda x_{1}+(1-\lambda)^{x_{2}}\right)+\mathrm{P}\right.$, called $f$ function it's convex.

When $\mathrm{Z}=\mathrm{R}, \mathrm{P}=[0,+\infty], \quad f$ is the usual convex function.

Theorem 1.1 (Ky Fan intercept theorem ) ([2]) Set K to Hausdorff Topological linear space X a non-empty compact convex set on the, and set $\mathrm{L}$ as $\mathrm{K} \times \mathrm{K}$ subset of the following properties:

(a) $\forall x \in K, \quad(x, x) \in \mathrm{L}$;

(b) $\forall y \in K$, Collection $\mathrm{L}\left({ }^{y}\right)=\{x \in K$ : $(x, y) \in \mathrm{L}\}$ in $\mathrm{K}$ the is a closed set;

(c) $\forall x \in K$, Collection $\mathrm{M}(x)=\{y \in K$ : $(x, y) \notin \mathrm{L}\}$ convex;

The $x_{0} \in \mathrm{K}$, makes $\left\{{ }^{X_{0}}\right\} \times \mathrm{K} \subset \mathrm{L}$.

\section{Primary Results}

Theorem 2.1 Set $K$ to Hausdorff a non-empty compact subset on a topological linear space, $P$ to Hausdorff topological linear space $\mathrm{Z}$ a closed convex cone in the, and int $P \neq \phi$. Set $f: K \times K \rightarrow Z$ meet the conditions:

(I) $\forall x \in K, f(x, x) \in P$;

(II) $\forall x \in K$, Mapping $y \rightarrow f(x, y)$ is convex;

(III) $\forall y \in K$, Collection $\{x \in K: f(x, y) \notin-\text { int } P\}_{\text {in }} \quad K \quad$ the is closed.

The $\exists X_{0} \in \mathrm{K}$ makes $f\left(x_{0}, y\right) \notin-$ int $P, \quad \forall y \in K$.

Proof: Order $L=\{(x, y) \in K \times K: f(x, y) \notin-$ int $P\}$,by criteria ( a ), $\forall x \in K, \quad f(x, x) \notin$-int $P$, $\because P \cap(-$ int $P)=\phi, \therefore f(x, x) \notin-$ int $P$.

So $(x, x) \in L, L$ is an empty set.

Also by the conditions $\left({ }_{\mathrm{C}}\right), \forall y \in K$, Collection $\{x \in K: f(x, y) \notin$-int $P\}$ in $K$ is closed, The collection $L\left({ }^{y}\right)=\{x \in K:(x, y) \in L\}$

$$
=\{y \in K: f(x, y) \notin \text {-int } P\}(\forall y \in K) \text { is a closed set. }
$$

$$
\begin{aligned}
\forall x \in K \text {, the collection } \mathrm{M}(x)=\{x \in K:(x, y) \notin L\} \\
=\{y \in K: f(x, y) \in-\text { int } P\} .
\end{aligned}
$$

under the $\mathrm{M}(x)$ is a convex subset.

In fact, $y_{1}, y_{2} \in \mathrm{M}\left({ }^{x}\right), t \in(0,1)$ and $z=t y_{1}+(1-t) y_{2}$ by Criteria (b), and note $\mathrm{P}$ for closed convex cone, available $f(x, z) \in t f\left(x, y_{1}\right)+(1-t) f\left(x, y_{2}\right)-P \in-$ int $P-P=-$ int $P$,

That $Z \in \mathrm{M}\left({ }^{X}\right), \mathrm{M}\left({ }^{X}\right)$ is convex. by Ky Fan intercept theorem, available: $\exists X^{X} 0 \in \mathrm{K}$ makes $\begin{cases}X & 0\end{cases}$ $\times \mathrm{K} \subset \mathrm{L}$,

That: $f\left(x_{0, y)} \notin\right.$-intP, $\forall y \in K$.

Theorem 2.2 Set $K$ to Hausdorff a non-empty compact subset on a topological linear space, $P$ to Hausdorff topological linear space $\mathrm{Z}$ a closed convex cone in the, and int $P \neq \phi$.set $f: K \times K \rightarrow Z$ meet the conditions: 
(a) $\forall x \in K, f(x, x) \in P$;

(b) $\forall x \in K$, mapping $y \rightarrow f(x, y)$ is convex;

$(c)^{\prime}: \forall y \in K, f(x, y)$ about $x$ continuous,

The $\exists x_{0} \in K$ makes $f(x, y) \notin$-intP,$\forall y \in K$.

Proof: by theorem 2.1 , we know that at this point, just by proving the criteria $(c)^{\prime}$ available conditions ${ }^{(c)}$, get the theorem 2.2 set up.

Conditions of proof below $(c)$ established, $\forall y \in K$, collection in $\{x \in K: f(x, y) \notin$-int P $\mathrm{K}$ is closed.

In fact, $\forall y \in K$, if the net $\left\{{ }^{x_{\alpha}}\right\} \subset\{x \in K: f(x, y) \notin-\operatorname{int} P\}$, and $x_{\alpha} \rightarrow^{x_{0}}$, to permit $x_{0} \in$ $\{x \in \mathrm{K}: f(x, y) \notin$-intP $\}$, cause $f(x, y)$ about $x$ continuous, there are $f\left(x_{\alpha}, y\right) \rightarrow f\left(x_{0}, y\right)$. $\because f\left(x_{\alpha}, y\right) \in \mathrm{Z} \backslash\{-\mathrm{intP}\}$, and $\mathrm{Z} \backslash\{-$ intP $\}$ the is a closed set.

$\therefore f\left(x_{\alpha}, y\right) \rightarrow f\left(x_{0}, y\right) \in \mathrm{Z} \backslash\{-\mathrm{intP}\}$, that $\square f\left(x_{0}, y\right) \notin$-int $\mathrm{P}$. so $x_{0} \in\{x \in \mathrm{K}: f(x, y) \notin$ - intP $\}$.

Inference 2.3 Set $\mathrm{K}$ to Hausdorff topological linear space $\mathrm{X}$ the last non-empty tight convex subset, $\mathrm{P}$ to Hausdorff topological linear space $\mathrm{Z}$ a closed convex cone in the, and int $\mathrm{P} \neq \phi$. set map, $T: K \rightarrow L(X, Z), \theta: K \times K \rightarrow X, \eta: K \times K \rightarrow Z$ meet the conditions:

(1) $\forall x \in K,<T x, \theta(x, x)_{>+} \eta(x, x) \in \mathrm{P}$;

(2) $\forall x \in K$,mapping $\mathrm{y}<\mathrm{Tx}, \theta(y, x)_{>+} \eta(x, y)$ about $y \in K$ is convex.

(3) $\forall x \in K$,collection $\left\{x \in K:<T x, \theta(y, x)_{>+} \eta(x, y) \notin-\right.$ int $\left.\mathrm{P}\right\}$ in $\mathrm{K}$ is closed.

Then (GVVI) has a solution, namely: $\exists x_{0} \in \mathrm{K}$ makes $<\mathrm{T}^{x}{ }_{0}, \theta\left(y, x_{0}\right)_{>+} \eta\left(x_{0} y\right) \notin$-intP, $\forall y \in K$.

Proof: in theorem 2.1 , take $f(x, y)_{=<\mathrm{T}} x, \theta(y, x)_{>+} \eta(x, y), \forall x, y \in K$. by the theorem 2.1 The is informed that the conclusion is correct.

Inference 2.4 Set K, X , P , Z , T , $\theta, \eta$ as inference 2.3 , and Conditions ( a ), ( b ) is established. also set the following conditions set up: (c) $\forall y \in K$, mapping $\quad x^{\mapsto}\langle T x, \theta(y, x)\rangle+$ $\eta(x, y)$ continuous.

Then (GVVI) has a solution.

Proof: in the theorem 2.2 , fetching $f(x, y)_{=<\mathrm{T}} x, \theta(y, x)_{>+} \eta(x, y), \forall x, y \in K$.by theorem 2.2 the concludes.

\section{Summary}

Well known, equilibrium problem is a meaningful generalization of variational inequalities and complementary problems, the is an important research topic in nonlinear analysis. Due to many problems such as mechanics, cybernetics, differential equations, mathematical economy and optimization theory, it can be summed up as equilibrium problem, so the research of this paper has a wide application background.

\section{References}

[1] Liu Bingyan, generic analysis, Science press, 2003, Beijing.

[2] Zhang Shiseng, variational inequalities and phase-complement problems (theory and application), Shanghai Science and Technology Press, (1991 Year, Shanghai). 
[3] Wang Yaodong, variational inequality equation, higher education publishing house, 1987 Year, Beijing.

[4] F. giannessi, theorems of alternative quadratic programs and complemtarity Prolems, in "variational in Equalities and C Omplementarity Problems ", ed.by R. w. cottle, f. giannessi and J. l. lions, j. wiley and Sons, New york,1980,151 186.

[5] F. giannessi (Ed), vector variational inequalities and vector equilibria, mathematical theories. Kluwer acad. publi. dordrecht, boston, london,2000. 